Trieste

\title{
DUE TOPONIMI COSTIERI ISTRIANI, BARBARIGA E BARABIGA
}

0 . Avranno pazienza i lettori se ancora una volta in questa Rivista - e con ciò ritengo anche di far cosa gradita al valoroso collega e amico Pavao Tekavčić - mi soffermo sull'analisi di due toponimi costieri istriani, precisamente Barbariga e $B a$ rabiga, toponimi i quali, sebbene designino due oggetti geografici distinti e lontani l'un l'altro, abbiano quindi storia e tradizione fra loro indipendenti, risalgono senz'altro - a mio modesto parere - ad uno stesso etimo. Ma vediamo un po', per ordine, come stiamo con la documentazione.

1. Barbariga è il nome, piuttosto noto, di una punta della costa occidentale istriana a metà strada circa tra Rovigno e Fasana, punta detta anche Cissana (o Cisana o Cesana oppure Pisana). A differenza di Barbariga questo Cissana è denominazione data alle volte, soprattutto nel passato (ad es. in Prospero Petronio), anche ad una punta più settentrionale, chiamata solitamente Punta San Paolo, qualche volta erroneamente Punta Gustigna (cfr. A. Šonje, ACRSR 11, 1980-81, p. 125); è quindi da presumere che questa singolare ambivalenza denominativa nasca dal fatto che Cissana fu denominazione, un tempo, di tutta la fascia costiera che si protende nell'Adriatico tra le due suddette punte e che solamente in epoca più vicina a noi si cercasse di tenere ben distinti i due capi che la delimitano, Punta San Paolo (erroneo Gustigna), appunto, a Nord e Barbariga a Sud. Non mi dilungherò troppo in questa sede sulla denominazione Cissana, che costituisce, dato il suo riferimento, presunto o reale, con la quasi mitica località istriana di Cissa, un problema a sé ed anche piuttosto aggrovigliato (a causa dell'omonimia con la località dalmatica, sull'isola di Pago, di Cissa o Gissa). Mi limiterò, piuttosto, a sottolineare che questo nome ha tutta l'aria di essere un prediale in -ANUM (o meglio in -ANA), forse da CAESIUS, e che proprio per questo motivo esso è stato esteso ad un'area piuttosto ampia. Aggiungerò, ancora, a riprova di questo possibile etimo, che nella zona sono stati scoperti anche i resti di una villa rustica romana (S. Zucchi, AMSIA 91, 1991, p. 294). ${ }^{1}$ Ad ogni modo anche Barbariga è denominazione, almeno oggi, piuttosto estesa, in quanto che, oltre la summenzionata punta, esiste anche una Stanzia Barbariga (cfr. VP 25-10-1985, p. 4) e volentieri si parla, in ambito turistico, di un insediamento Barbariga-Mandriol (VP 24-1-1983 ecc.). ${ }^{2}$

1 Su insediamenti paleocristiani nell'area adiacente (abbazia di Sant'Andrea) v. G. Cuscito "Il Piccolo" 15-6-1985).

2 Mandriol (già Mandariol) è località lungo la Via Flavia, dopo la chiesa di S. Martino e presso la Punta 
Rammentiamo ancora che esiste, accanto alla forma "standard" Barbariga, anche Barberiga (Carta geografica C. Salmon, a. 1703, cfr. Pellizzer p. 58). Ambedue, ad ogni modo, vanno considerate forme italianeggianti (o meglio venezianeggianti), in netta opposizione alla forma istriota che suona Barbaréiga (cfr. G. Curto in "Concorso Istria Nobilissima" XIV, Trieste-Fiume 1984, p. 135 e P. Tekavčić "Čak. Rev." XVI, 1988, p. 95). La forma italianeggiante è accettata anche nella toponomastica del "registro" croato (es. kod Barbarige B. Marušić in "Arheološka Istraživanja u Istri ecc.", Pula 1987, p. 91), mentre sarà certo un lapsus il Bargariga (sic) di A. Mirković "Concorso Istria Nobilissima" XVI, Trieste-Fiume 1983, p. 223, e, possibilmente anche il Barabéiga di G. Pellizzer p. 48 (in netto contrasto con il Barbaréiga di due pagine prima!). Di ben altro tenore, rispetto a queste due ultime "varianti", la dizione impiegata dal Coppo nel suo Portolano (a. 1528, cfr. Lago-Rossit Le "tabulae" I, Trieste 1986, p. 353) ualbarica, ossia Valbarica. Questo Valbarica, proprio perché non sospettabile di contaminazione con altro toponimo è, diversamente da quanto ricavabile dal Barabéiga del Pellizzer, lezione indubbiamente originale (quindi da tenersi nel massimo conto nell'analisi etimologica di questi nostri toponimi).

3. Barabiga è, invece, denominazione di una punta, di un seno di mare (valle) e di un prato in declivio ad esso prospiciente (ed esposto a mezzogiorno), tutti localizzati a N. di Rovigno, ai margini settentrionali dell'insenatura maggiore di Valdibora (dial. Valdabora-Maldabora). Si tratta di una denominazione di tradizione piuttosto recente: la più antica è quella della Carta Milit. Austriaca (Rilievi a. 1894), cui segue quella dello Jurišić (1956), p. 10, nonché quella del Portolano del Mediterraneo vol. VI $\left({ }^{2}, 1972 / 85\right)$ p. 81 , il quale riporta la dizione "Rt Barabiga". Si confronti anche G. Giuricin "Il Piccolo" 7-5-1975, Al. Cella AMSIA 81 (1981) p. 18 (e di nuovo G. Giuricin, "Voce della Famia Ruvignisa", N. 41, ge.-fe. 1989, p. 47, n. 1, il quale ammonisce a non confondere Barabiga con Barbariga). Il toponimo, nel registro istriotizzante, suona Barabéiga, come annota il Pellizzer, pp. 28-34, il quale Pellizzer a sua volta tiene a distinguere tra Ponta da Barabéiga e sottostante Valistréin da Barabéiga (distinzione già operata dallo Jurišić). Nella carta dell' IGM Barabiga non compare, la punta è chiamata col nome alternante di Punta Figarola o della Cava e la relativa insenatura è senza nome. D'altro canto la stessa comporta, in dialetto rovignese, anche un altro nome, Val da lisso ("lo specchio d'acqua di fronte ai prati di Barabiga", così la testimonanza di un vecchio pescatore, PV 29-11-1991-p.5, la quale serve nello stesso tempo anche come attestazione del terzo impiego - v. sopra - di Barabiga), nel registro venezianeggiante Val de Lesso (VP 14-10-1982, ecc.) o anche Valle del Lèsso (TSO 4-12-1991), cr. Uvala Lisso ${ }^{3}$, località venuta piuttosto recentemente alla ribalta della cronaca, causa il nome del vicino camping, Kamp Beograd o Campo Belgrado, al quale un gruppo di profughi da Ragusa non volle accedere se non

Barbariga, cfr. B. Benussi, Geografia dell'Istria (Trieste 1877), p. 118.

3 Si parte, a dir il vero, da voci dialettali locali significanti "leccio" (cfr. presso Pola Monte Lesso, anticamente [Schiavuzzi] Monte Lezze), da lat. ILǏCIUM (per gentile suggerimento della laureanda sig. na Samantha Rocco, di Rovigno). 
si fosse provveduto immediatamente a ripristinare il nome locale originario. Val da Lisso per l'appunto.

La situazione denominativa relativa a Barabiga/Barabéiga si presenterebbe, così, abbastanza omogenea, più di quanto non sia per Barbariga/Barbaréiga, se nel Portolano del Mediterraneo del 1927 non comparisse inaspettata e piuttosto disturbante l'isolata dizione Punta Barbariga. Come giudicarla? Lapsus per Barabiga (dovuto ad una banale confusione con il più noto Barbariga "Punta Cissana"), o forma alternante di Barabiga, quindi in qualche modo originale, per lo meno rappresentante di un usus linguistico particolare? Al momento attuale dello status della documentazione non possiamo decidere. Conviene, perciò, ai fini della discussione etimologica che fra breve seguirà, non utilizzarla, anche se questo Barbariga al posto da Barabiga (così come il Barabéiga del Pellizzer al posto di Barbariga) ci tornerebbe, in ultima analisi, assai comodo.

4.1. Etimi finora proposti. Dato lo scarso spessore cronologico in cui si dispongono le attestazioni di Barabiga, lo sforzo, se così possiamo chiamarlo, etimologico si è concentrato su Barbariga.

L'etimo più convincente sembrerebbe quello per cui si partirebbe da un nome di famiglia o Barbarighi (i quali avrebbero posseduto dei terreni in quei pressi, G. Fabro in "Dignano e la sua gente"; Trieste 1975, p. 121) o Barbariga (Rosamani s.v.); A. Coglievina ("Arena di Pola" 22-7-1989, p. 4) sottolinea a questo proposito che una famiglia Barbariga viveva in quel di Padova, precisamente a Vigonza, e a questo punto si innesta anche la possibilità che cotesto Barbariga si possa raccostare in qualche modo con Barbarigo, bastione della cinta fortificata di Curzola (G. Ziliotto R. Dalm. 5, 1985, p. 11). Ipotesi queste abbastanza suggestive dal momento che i toponimi molto spesso derivano da un personale (ma alle volte succede proprio l'opposto). Però altrettanto valida si presenta l'ipotesi anche di chi vedrebbe in Barbariga un prediale in -İCUM (tipo Lavarigo < LABERIUS), dal nome latino BARBARIUS o BARBARUS.

4.2. Sennonché in nessuna di queste proposte si tiene nel debito conto della testimonianza del Coppo, il quale, come si è già detto, ci offre la variante Valbarica. Di fronte a questo fatto non sussistono altre possibilità se non quella per cui il nostro Barbariga, derivi, insieme e Valbarica, da VALLIS APRICA. Non solo, ma VALLIS APIRICA è base accettabile per spiegare anche l'altro toponimo che ci sta a cuore, il rovign. Barabéiga. Attraverso semplici fenomeni di metatesi e/o sincopi dissimilative VALLIS APRİCA "valle soleggiata" passa a *VALBRICA e di qui a *Valbariga (che il Coppo modifica, lievemente italianeggiando, in *Valbarica) e a *Balbariga, da cui i nostri Barbariga (-éiga) da una parte e Barabiga (-éiga) dall'altra. In altre parole, Barbariga e Barabiga hanno lo stesso etimo, indipendentemente dal fatto che una volta sola compaia attestata per Barbariga la forma (Pellizzer) Barabéiga e per Barabiga la forma (Portolano 1927) Barbariga.

I supporti a questo nostro etimo sono tutti validi. 
Prima di tutto il passaggio di V iniziale a $\mathrm{B}$ è abbastanza diffuso nella nostra area (coste istriane e di Cherso-Lussino). Basti rammentare Baldarca di Sansego, parallelo a Valdàrika di Arbe e Valdarche di Lussino, evidentemente "Valle (=baia) dell'E RICA", poi Babiska, a Veglia, variante di Valbisca (Bartoli II 237), nonché Balvanida, insenatura sulla costa occidentale di Lussino, da Valbanida, ossia "valle bandita" (cfr. G. Maver RL 2, 1953, p. 185). E non è nemmeno da dimenticare la denominazione cr. Bale per Valle (d'Istria), da lat. VALLIS.

In secondo luogo APRICUS "solatio" non può definirsi estraneo alla toponomastica né italiana né di aree contermini. Ricorderemo il toponimo lig. L'abrigasu (presso Pigna) "pendio erboso rivolto a mezzogiorno" (LEI III, 19, 1985, c. 360 [Fazio-Pfister]), nonché il più noto colle dell'Aprica (dial. l'abriga, l'avriga, la vriga) in provincia di Sondrio (Dizionario di toponomastica U.T.E.T., Torino 1990, p. 53 [C. Marcato]). Nel dalmatico APRĪCUS sta certo alla base del toponimo Japirk (presso Salona), a sua volta connesso con cr. jâprk"solatio" (a. 1550, V. Vinja RLIR 21, 1957, p. 51. P Skok ERHiSJ II s.v., ecc: scettico comunque L. Rocchi p. 48), possibilmente da APRİCUM incrociatosi con AFRICCUM. Ma questo non è l'unico caso del genere: proprio lungo la costa istriana occidentale esiste un toponimo ben neto Àbrega (nome di centro abitato e anche di insenatura, a N. di Parenzo) con testimonianze a partire dal 1530 (Abriga) e comportante anche forme del registro croato lievemente divergenti ma molto sintomatiche tipo Òbriga (a. 1806), Vabriga o Vobriga (dove V-rappresenta, come spesso, l'agglutinazione della preposizione locativale slava $v$ o $u$ ). Preciseremo che forse non tanto l'incrocio con AFRĬCUS (cfr. F. Crevatin PIstr. 1979 f. 4, p. 35) quanto il passaggio alla forma dialettale croata abbia favorito lo spostamento d'accento.

Tutto ciò (altro argomento), in netta opposizione al talasson. Valdabora (o Val di bora), il quale pone in rilevo l'esposizione a Nord, al vento di Tramontana (o di Bora), del resto dell'insenatura rovignese. Ma anche per Barbariga valgono le stesse osservazioni, qualora detta punta la si giudichi nella sua posizione con riferimento alla sottostante insenatura di Val Casematte (v. IGM).

In terzo luogo non va sottaciuto, a parer mio, nemmeno il fatto che l'etimo immediato da un sintagma con valore di appellativo ("valle aprica"), anziché da altro nome proprio (famiglia Barbarighi ecc.), potrebbe essere comprovato dal fatto che talora detto toponimo, al pari del lombardo l'Aprica, richieda l'uso dell'articolo determinativo, es. "La Barbariga", G. Rieger Costa occid. d'Istria (1845) r. 50, "fino alla vecchia Barbariga" VP 9-7-1988, p. 5.

Infine, e questa volta con riferimento unicamente ai Realien, rammentiamo che la denominazione rovignese Barabiga si applica, come del resto abbiamo già accennato, non solo ad uno specchio d'acqua ma ad un prato in pendio lungo la costa stessa, quindi, dato l'orientamento del sito, esposto effettivamente a mezzogiorno. 


\section{ABBREVIAZIONI IMPIEGATE}

ACRSR “Atti del Centro di Ricerche Storiche di Rovigno, Trieste-Fiume."

AMSIA "Atti a Memorie della Società Istriana di Archeologia e Storia Patria," Parenzo e poi Trieste.

Bartoli Matteo Bartoli, Das Dalmatische, Wien 1906.

Jurišić Blas Jurišić, Toponimika zapadne Istre, Cresa i Lošinja, Anali, Leksikografski Zavod FNRJ 3, Zagreb 1955.

LEI "Lessico Etimologico Italiano", a cura di M. Pfister, Wiesbaden 1979.

Pellizzer Giovanni Pellizzer, Toponomastica della costa rovignese, Settala 1975.

PIstr. "Pagine Istriane". Organo dell'Associazione Istriana di Studi e Storia Patria, Parenzo, poi Trieste.

RDalm. "Rivista Dalmatica", Associazione Nazionale Dalmata, Roma.

RL

"Ricerche Linguistiche", Istituto di Glottologia, Università di Roma.

RLiR

"Revue de linguistique Romane", Paris.

Rocchi Luciano Rocchi, Latinismi e romanismi antichi nelle lingue slave meridionali, Udine 1990.

Rosamani Enrico Rosamani, Vocabolario Giuliano, Bologna 1958 (rist. 1991).

Skok ERHiSJ Petar Skok, Etimologijski rječnik hrvatskoga ili srpskoga jezika, Zagreb 1971-74.

TSO

"Trieste Oggi. Giornale della Città."

VP

"La Voce del Popolo", Fiume.

Povzetek

DVE KRAJEVNI IMENI Z ISTRSKE OBALE: BARBARIGA IN BARABIGA

Dva kraja na istrski obali, Barbariga, na pol pota med Rovinjem in Fažano, in Barabiga, majhen rt severno od Rovinja ob zalivu Valdabora/Maldabora, imata po avtorjevem mnenju isti etimološki vir. Večina razlagalcev je enotna $v$ prepričanju, da je treba iskati vir za Barbarigo $v$ imenu družine Barbarigov. Avtor pa opozarja na manj znano, a zapisano različico krajevnega imena Valbarica, kar gotovo izhaja iz latinskega VALLIS APRICA 'osončena, $k$ soncu obrnjena dolina'; izbrano ime se lepo sklada $z$ lego enega in drugega kraja. $\mathrm{V}$ prispevku se navaja $\mathrm{v}$ potrdilo take razlage vrsta toponimov, ki imajo $\mathrm{v}$ sebi latinski APRICUS. Tudi nekateri hrvaški kraji, npr. Japirk pri Solinu.

Razlago podpira tudi raba določnega člena v italijanskih virih: La Barbariga. Ta je prav naravna ob občnem imenu, je pa vsaj močno presenetljiva ob lastnem imenu. 